\title{
Ecologia e ontogenia da alimentação de Astyanax janeiroensis (Osteichthyes, Characidae) de um riacho costeiro do Sudeste do Brasil
}

\author{
Rosana Mazzoni ${ }^{1,3}$, Livia Lourenço Nery ${ }^{1}$ \& Ricardo Iglesias-Rios ${ }^{2}$ \\ ${ }^{1}$ Laboratório de Ecologia de Peixes, Departamento de Ecologia, Universidade do Estado do Rio de Janeiro - UERJ, \\ Av. São Francisco Xavier, 524, CEP 20550-011, Maracanã, RJ, Brasil \\ ${ }^{2}$ Departamento de Ecologia, Instituto de Biologia, Universidade Federal do Rio de Janeiro - UFRJ, \\ CP 68020, Rio de Janeiro, RJ, Brasil \\ ${ }^{3}$ Autor para correspondência: Rosana Mazzoni,e-mail:mazzoni@uerj.br
}

MAZZONI, R., NERY, L., IGLESIAS, R.I. Ecology and ontogeny of feeding habit of Astyanax janeiroensis (Osteichthyes, Characidae) from a coastal stream from Southeast Brazil. Biota Neotrop. 10(3): http://www. biotaneotropica.org.br/v10n3/en/abstract?article+bn01010032010.

\begin{abstract}
In the present work we aimed to describe the spatio-temporal and ontogenetic variations of Astyanax janeiroensis diet in Ubatiba stream, a coastal fluvial system from Serra do Mar. We analyzed 540 specimens collected monthly during twelve months in six sites differing in the degree of vegetal cover (opened and closed sites). We verified that $A$. janeiroensis is an omnivorous species whose diet is largely based on autochthonous items. The estimated intestinal coefficient was $0.74(+1.2)$, being compatible with an omnivorous behavior. We did not register differences in the diet from dry and rainy seasons. Nonetheless, we found differences in the diet from opened and closed sites. We registered quite absolute predominance of autochthonous items in the diet from the closed sites, whereas, in the opened ones we found similar ingestion of alo and autochthonous items. Concerning juveniles and adult specimens, we found differences in the consumption of animal and vegetal items with a relative reduction of animal ingestion among adult specimens. The intestinal coefficient of adult specimens was higher than that of juveniles, corroborating the diet results.
\end{abstract}

Keywords: feeding, ontogeny, Characiforms, omnivorous.

MAZZONI, R. NERY, L. IGLESIAS, R.I. Ecologia e ontogenia da alimentação de Astyanax janeiroensis (Osteichthyes, Characidae) de um riacho costeiro do Sudeste do Brasil. Biota Neotrop. 10(3): http://www. biotaneotropica.org.br/v10n3/pt/abstract?article+bn01010032010.

Resumo: No presente trabalho tivemos como objetivo descrever as variações espaço-temporais e ontogenéticas da dieta de Astyanax janeiroensis do Rio Ubatiba, um riacho costeiro da Serra do Mar. Analisamos o conteúdo gástrico de 540 exemplares capturados mensalmente, durante 12 meses, em seis localidades, sendo três com cobertura (localidade fechada) vegetal e três sem cobertura (localidade aberta). Verificamos que A. janeiroensis é uma espécie onívora, com dieta essencialmente baseada em itens autóctones, majoritariamente de origem vegetal. O coeficiente intestinal da espécie foi estimado em $0,74(+1,2)$ que é um valor compatível com o hábito alimentar onívoro. Não foram registradas diferenças na dieta dos exemplares coletados durantes as estações seca e chuvosa. No entanto, foram registradas diferenças na dieta dos exemplares coletados nas localidades aberta e fechada. Nas localidades fechadas houve predominância quase absoluta de itens autóctones, enquanto nas localidades abertas houve participação equivalente dos itens alóctones e autóctones. Registramos que os exemplares jovens e adultos diferem no consumo dos itens animais e vegetais com redução relativa do consumo e animais entre os adultos. Os valores do coeficiente intestinal dos jovens foram superiores aos dos adultos, corroborando os resultados da dieta.

Palavras-chave: alimentação, ontogenia, Characiformes, omnivoro. 


\section{Introdução}

Diversos registros da literatura sobre os hábitos alimentares de peixes de riacho têm demonstrado importante dependência da floresta circundante como fonte de importação dos recursos alimentares (e.g. Costa et al. 1987, Aranha et al. 1998, Abilhoa et al. 2007, Dufech et al. 2003, Rezende \& Mazzoni 2006a, b). De fato, diversas espécies de peixes têm sua dieta amplamente baseada em recursos de origem alóctone (Lowe-McConnell, 1991) e alterações na vegetação ripária mostraram ter efeito direto na dinâmica da alimentação dessas espécies de riacho (Casatti 2002, Rezende \& Mazzoni 2006b). No entanto, variações na dieta também podem ser causadas por outros fatores, tais como desenvolvimento ontogenético (Gerking 1994), maturação sexual (Barbieri et al. 1994) ou fase do ciclo reprodutivo (Wootton 1998). Essas mudanças são, normalmente, acompanhadas de alterações morfológicas, preferência por diferentes tipos de recurso trófico e exploração de hábitats (Wootton 1998, Matthews 1998). A despeito das alterações morfológicas e comportamentais que acompanham o desenvolvimento, vários estudos mostraram claramente que os peixes tropicais exploram uma ampla gama de itens alimentares, mas em função da estrutura e comprimento do ducto gastrointestinal, existe um limite pré-estabelecido para a variabilidade da alimentação (e.g. Angelescu \& Gneri 1949, Lowe-McConnell 1991, Pringle \& Hamazaki 1998).

A família Characidae compreende um grupo de peixes muito heterogêneo e está organizada em diversos gêneros incertae sedis (Lima et al. 2003). Por sua extrema variabilidade é o grupo mais bem sucedido e diversificado, lhe permitindo ocupar diferentes hábitats, tanto lóticos como lênticos, e desenvolver variadas estratégias alimentares (Mazzoni et al. 2004). Astyanax janeiroensis Eigenmann, 1908 pertence à família Characidae, apresenta distribuição restrita aos riachos costeiros do Leste do Brasil e tem ampla distribuição ao longo no Rio Ubatiba, área de estudo do presente trabalho (Mazzoni \& Lobón-Cervià 2000).

Estudos realizados sobre a alimentação de diferentes espécies de Characidae mostraram que as espécies desse gênero costumam se alimentar tanto de itens de origem vegetal como animal e que, portanto, têm dieta onívora. Porém, vários relatos sobre a alimentação de espécies de Characidae têm demonstrado a existência de alterações importantes relacionadas ao crescimento (Rezende \& Mazzoni 2006a, b, Mazzoni \& Costa 2007). Neste trabalho tivemos como objetivo descrever as variações ontogenéticas da dieta de Astyanax janeiroensi bem com suas variações espaço-temporais decorrentes das estações seca e chuvosa e do grau de cobertura vegetal das diferentes localidades de estudo.

\section{Material e Métodos}

\section{1. Área de estudo}

O Rio Ubatiba ( $22^{\circ} 60^{\prime} \mathrm{S}$ e $42^{\circ} 48^{\prime} \mathrm{W}$ ) compõe um pequeno sistema fluvial que drena a vertente ocidental da Serra do Mar no Estado do Rio de Janeiro. Sua drenagem é regulada essencialmente pelas chuvas (c. $1500 \mathrm{~mm} . \mathrm{ano}^{-1}$ ) com aumento significativo da vazante durante a época de verão (novembro - janeiro) (Mazzoni \& Lobón-Cervià 2000). Não obstante o padrão sazonal resultante das chuvas de verão, chuvas torrenciais imprevisíveis (> $120 \mathrm{~mm} \cdot \mathrm{dia}^{-1}$ ) são frequentes ao longo de todo o ano. A ictiofauna do Rio Ubatiba é composta por 22 espécies (Mazzoni et al. 2006) que compreendem 7,4\% do total de peixes de água doce que ocorrem nos riachos costeiros do Leste do Brasil. A caracterização fisiográfica baseada nos parâmetros físico-químicos de condutividade, tipo de substrato e tipo de corrente e biológicos de grau de cobertura vegetal e abundância de vegetação marginal aquática é apresentada para cada uma das localidades amostradas (Tabela 1) e, conforme mencionado em Mazzoni \& Lobón-Cervià (2000) não apresentam um padrão longitudinal de mudança, mas sim uma distribuição em mosaico, com todos os tipos de mesohábitat compondo a estrutura física de cada localidade. A vegetação de cobertura é o único parâmetro que, efetivamente, muda ao longo do riacho.

\section{Amostragem}

Coletas mensais, por pesca elétrica (CA, 220V - 2-3A; Mazzoni et al. 2000), foram realizadas, entre dezembro/2004 e novembro/2005, em seis localidades ao longo do eixo longitudinal do Rio Ubatiba. As localidades de estudo se distinguiam pelo grau de cobertura vegetal e caracterizaram dois tipos distintos de ambientes: (i) localidade aberta e (ii) localidade fechada (Figura 1). A cada ocasião de amostragem eram coletados entre 30 e 50 exemplares de Astyanax janeiroensis (Figura 2). Os exemplares amostrados eram mantidos em gelo para transporte e, em laboratório, processados para registro dos dados de Comprimento padrão $(\mathrm{Cp}, \mathrm{cm})$ e Peso total $(\mathrm{Pt}$, g). Posteriormente, eram dissecados para retirada do trato digestivo e determinação do Peso do estômago (Pe, gr) e Comprimento do intestino $(\mathrm{Ci}, \mathrm{cm})$. Cada estômago processado era fixado em formol $5 \%$ por 3 dias e, posteriormente, conservado em álcool etílico $70{ }^{\circ} \mathrm{GL}$.

\section{Tratamento dos dados}

A análise do conteúdo estomacal foi realizada através dos métodos Volumétrico (Vo) e da Frequência de Ocorrência (Fo) (Hyslop, 1980) mediante microscópio estereoscópico. Os ítens alimentares foram identificados, ao nível de ordem, de acordo com

Tabela 1. Valores médios (Média) e desvio padrão (dp) dos parâmetros de condutividade $(\mathrm{C}-\mathrm{nS})$, largura aferida em metros $(\mathrm{L}-\mathrm{m})$, profundidade máxima aferida em centímetros (PMax - cm), tipo de corrente (C - poças, corredeiras -Cor e rápidos -Rap), tipo de substrato (areia -Are, cascalho -Cas e seixos -Seix) e área amostrada padronizada para metros quadrados $\left(\mathrm{Ar}-\mathrm{m}^{2}\right)$, registrados para as duas estações de coleta (seca e chuva) a partir de transectos transversais, com distância de $5 \mathrm{~m}$ entre eles, nas seis localidades estudadas no sistema fluvial do Rio Ubatiba.

Table 1. Mean (Média) and standard deviation (dp) values for data of conductivity $(\mathrm{C}-\mathrm{nS})$, width in meters $(\mathrm{L}-\mathrm{m})$, maximum deep in centimeters $(\mathrm{PMax}-\mathrm{cm})$, current (Corrente - pools, run - Cor and rapids - Rap), substratum (sand -Are, gravel - Cas e coubles -Seix) and sampled area $\left(\mathrm{Ar}-\mathrm{m}^{2}\right)$, registered for each season (dry and rainny seasons) based on transects made from $5 \mathrm{~m}$ each one, at each of the six study sites in Ubatiba Stream.

\begin{tabular}{|c|c|c|c|c|c|c|c|c|c|c|c|c|c|c|}
\hline \multirow[t]{2}{*}{ Locs } & \multicolumn{2}{|c|}{$C-\mu S$} & \multicolumn{2}{|c|}{$\mathbf{L}-\mathbf{m}$} & \multicolumn{2}{|c|}{ PMax - cm } & \multicolumn{3}{|c|}{ Corrente } & \multicolumn{3}{|c|}{ Substrato } & \multicolumn{2}{|c|}{$A r-m^{2}$} \\
\hline & Média & dp & Média & Dp & Média & dp & Poça & Cor & Rap & Are & Casc & Seix & Média & dp \\
\hline U1 & 80 & 5 & 2,2 & 0,8 & 48,7 & 13,19 & 20,3 & 68,8 & 10,9 & 19,7 & 59,3 & 21,0 & 195,1 & 27,4 \\
\hline U2 & 90 & 8 & 1,5 & 0,7 & 40,1 & 8,9 & 14,9 & 47,7 & 37,4 & 16,7 & 73,3 & 10,0 & 135,3 & 36,1 \\
\hline U3 & 95 & 4 & 1,3 & 0,4 & 51,2 & 9,7 & 13,0 & 15,0 & 72,0 & 15,0 & 78,0 & 7,0 & - & - \\
\hline U4 & 110 & 10 & 1,7 & 0,9 & 49,6 & 12,8 & 25,6 & 60,9 & 13,5 & 49,9 & 21,3 & 28,8 & 109,1 & 34,2 \\
\hline U5 & 180 & 11 & 1,9 & 0,9 & 54,0 & 15,9 & 9,7 & 84,7 & 5,6 & 25,3 & 66,6 & 8,1 & 132,7 & 42,7 \\
\hline U6 & 380 & 20 & 2,1 & 0,9 & 45,7 & 11,6 & 8,0 & 74,7 & 17,3 & 58,3 & 34,9 & 6,8 & 114,4 & 29,6 \\
\hline
\end{tabular}



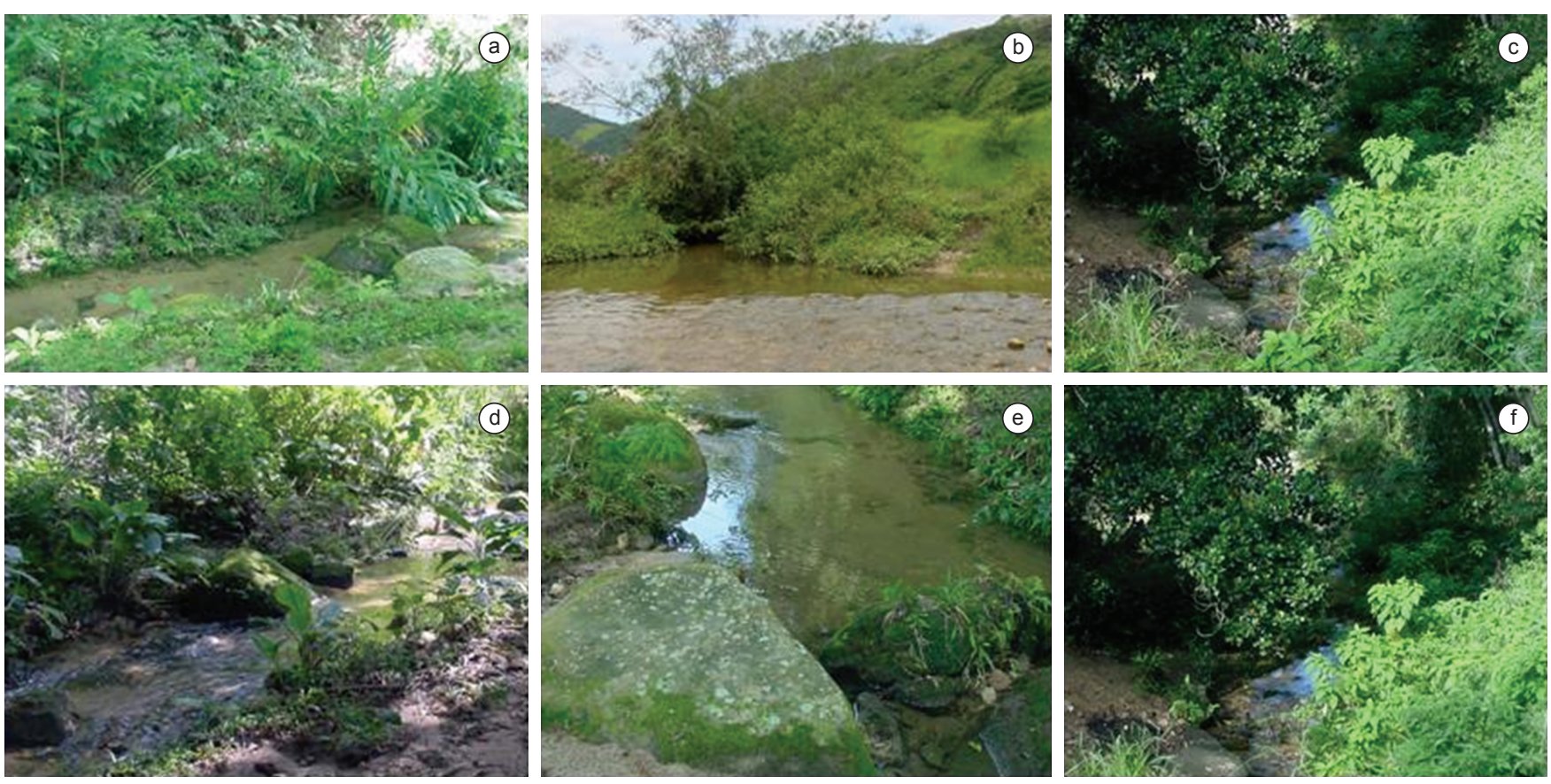

Figura 1. Localidades amostradas no Rio Ubatiba, a, b, e) representam as localidades sem cobertura vegetal (Abertas) e c, d, f) representam as localidades com cobertura vegetal (fechadas).

Figure 1. Study sites at Ubatiba Stream, a, b, e) show sites without vegetal cover (Opened) and c, d, f) show sites with vegetal cover (closed).

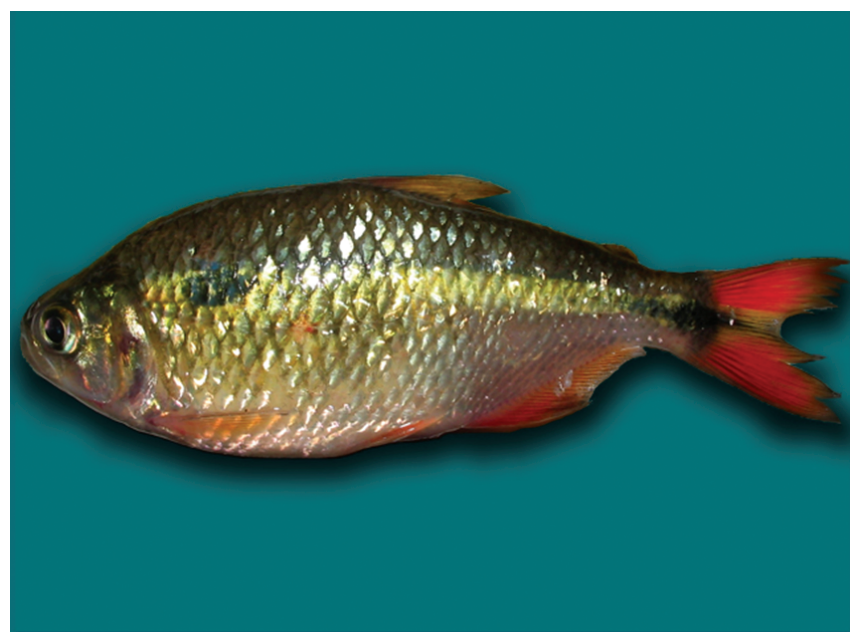

Figura 2. Exemplar de Astyanax janeiroensis (7,2 cm) amostrado no Rio Ubatiba.

Figure 2. Astyanax janeiroensis specimen $(7,2 \mathrm{~cm})$ sampled at Ubatiba Stream.

a literatura disponível (Carvalho, 1989, 1992, Carvalho et al. 2001, Melo 2003, Olifiers et al. 2004, Salles et al. 2004, Borror \& Delong 2005, Pes et al. 2005, Passos et al. 2007). A participação relativa de cada categoria registrada nos estômagos em relação à totalidade da dieta foi analisada através do Índice de Importância Alimentar (IA $)$ proposto por Kawakami \& Vazzoler (1980) e adaptado por Hahn et al. (1997). O IA foi aplicado de acordo com o modelo a seguir: $\mathrm{IA}_{\mathrm{i}}=\left(\mathrm{F}_{\mathrm{i}}^{*} \mathrm{~V}_{\mathrm{i}}\right) /\left(\sum \mathrm{F}_{\mathrm{i}}^{*} \mathrm{~V}_{\mathrm{i}}\right) * 100$, onde $\mathrm{i}=1,2, \ldots . \mathrm{n}$ categorias alimentares; $\mathrm{F}_{\mathrm{i}}=$ frequencia de ocorrência de determinada categoria alimentar; $\mathrm{V}_{\mathrm{i}}=$ volume de determinada categoria alimentar.

A análise dos itens utilizados foi baseada no somatório do IA de cada categoria alimentar (animal vs. vegetal) e origem (autóctone vs. alóctone). A fim de se avaliar possíveis variações ontogenéticas da dieta foi analisado o somatório do $\mathrm{IA}_{\mathrm{i}}$ das duas categorias alimentares para duas classes de tamanho (jovens e adultos), sendo estas determinadas com base no tamanho da primeira maturação (5,5 cm - Mazzoni et al. 2006). O Coeficiente Intestinal (CI) foi determinado de acordo com o modelo $\mathrm{CI}=\mathrm{Ci} / \mathrm{Cp}$ (sensu Barbieri et al. 1994). A aplicação desse modelo serviu como informação acessória para a classificação trófica de espécie e complementou a análise do hábito alimentar de jovens e adultos. A análise espaço temporal da dieta foi tratada com base nos dados de $\mathrm{IA}_{\mathrm{i}}$ das diferentes categorias e (animal e vegetal) e origens (alóctone e autóctone) obtidos durante as estações seca e chuvosa (temporal) em dois tipos de localidades que se distinguiram pelo grau de preservação da cobertura vegetal (espacial).

\section{Resultados}

Foram analisados 540 exemplares, sendo 259 jovens e 281 adultos. Verificamos que a dieta de A. janeiroensis é baseada tanto em itens de origem animal como vegetal, embora tenha-se registrado maior participação dos itens de origem vegetal (Figuras 3, 4 e 5). Entre os animais que compõem a dieta se destacam formas adultas de insetos de origem alóctone (Hymenoptera, Aranae e Isoptera) e formas adultas (Crustacea) e larvais (Coleoptera e Odonata) de artrópodos de origem autóctone; também foram encontrados ovos, escamas e peixes, sendo estes últimos registrados em uma ou poucas ocasiões. Dentre os itens de origem vegetal se destacam algas filamentosas, sementes e fragmentos vegetais alóctones oriundos da vegetação marginal semiaquática (Tabela 2 e 3). O coeficiente intestinal médio da espécie foi estimado em $0,74( \pm 1,2)$ indicando dieta onívora.

A análise do conteúdo gástrico, de jovens e adultos, apontou tendência para o aumento relativo da ingestão de itens de origem vegetal na medida em que há incremento no tamanho dos indivíduos (i.e. na medida em que atingem a maturação sexual). O IAi total analisado indicou que entre os exemplares jovens $42,4 \%$ dos itens consumidos eram animais e 57,6\% eram vegetais, enquanto entre 
os exemplares adultos $23,9 \%$ dos itens consumidos eram animais e $76,1 \%$ eram vegetais (Figura 3; Tabela 2). Os valores médios do CI de jovens $(0,64)$ e adultos $(0,84)$ apresentaram diferenças significativas $(\mathrm{t}=-2,72 ; \mathrm{GL}=52 ; \mathrm{p}=0,008)$ indicando que a relação entre o tamanho do intestino e o comprimento padrão de A. janeiroensis varia ao longo do crescimento dos indivíduos. Esse resultado foi corroborado pelos resultados da dieta de jovens e adultos, confirmando a tendência a redução relativa da carnivoria entre os exemplares adultos.

Não houve importantes diferenças entre as dietas das estações seca e chuvosa, tanto para o consumo dos itens de diferentes origens (alóctone e autóctone) como para as diferentes categorias (animal e vegetal). Registramos predomínio de itens vegetais de origem autóctone em ambas as estações, seca e chuvosa (Figura 4; Tabela 3). O padrão espacial do consumo dos diferentes itens alimentares evidenciou que os itens vegetais são consumidos em igual quantidade em ambas as localidades, porém os itens de origem alóctone predominam sobre os autóctones nas localidades abertas (Figura 5; Tabela 3).

Tabela 2. Dados da Frequência de Ocorrência (FO), Volume (VO) e Índice Alimentar (IAi) dos diferentes itens alimentares registrados na dieta de jovens e adultos de Astyanax janeiroensis amostrados no sistema fluvial do Rio Ubatiba. CI médio = Coeficiente Intestinal médio de exemplares jovens e adultos.

Table 2. Frequency of occurrence (FO), volume (VO) and Alimentary Index (IAi) of each food items registered in the diet young and adult specimens of Astyanax janeiroensis sampled at the opened and closed sites at Ubatiba Stream. Mean Intestinal Coefficient (CI médio).

\begin{tabular}{|c|c|c|c|c|c|c|}
\hline \multirow[t]{2}{*}{ Itens } & \multicolumn{3}{|c|}{ Jovens } & \multicolumn{3}{|c|}{ Adultos } \\
\hline & FO & VO & IAi & FO & VO & IAi \\
\hline \multicolumn{7}{|c|}{ Itens alóctones } \\
\hline \multicolumn{7}{|c|}{ Formas adultas de artropodas } \\
\hline Hymenoptera & 104 & 310 & 11,71 & 32 & 1678 & 7,63 \\
\hline Araneae & - & - & - & 2 & 6 & $<0,01$ \\
\hline Isóptera & - & - & - & 2 & 4 & $<0,01$ \\
\hline \multicolumn{7}{|c|}{ Matéria vegetal } \\
\hline Semente & 60 & 42 & 0,95 & 20 & 162 & 0,46 \\
\hline Restos vegetais & 210 & 638 & 48,18 & 74 & 6324 & 66,56 \\
\hline \multicolumn{7}{|c|}{ Itens autóctones } \\
\hline \multicolumn{7}{|c|}{ Formas jovens de artrópodos } \\
\hline Lepidoptera & 20 & 4 & 0,03 & 4 & 1054 & 0,60 \\
\hline Diptera & 80 & 108 & 3,26 & 12 & 28 & 0,05 \\
\hline Thricoptera & - & - & - & 2 & 6 & 0,00 \\
\hline Coleoptera & - & - & - & 14 & 1012 & 2,01 \\
\hline Hymenoptera & 20 & 4 & 0,03 & 8 & 48 & 0,05 \\
\hline Odonata & 20 & 60 & 0,45 & 10 & 774 & 1,10 \\
\hline \multicolumn{7}{|c|}{ Formas adultas de artropodas } \\
\hline Crustacea & 80 & 272 & 8,22 & 12 & 1074 & 1,83 \\
\hline Fragmento de insetos & 146 & 302 & 15,96 & 44 & 1516 & 9,47 \\
\hline Hemiptera & - & - & - & 8 & 80 & 0,09 \\
\hline Coleoptera & 26 & 6 & 0,05 & 12 & 82 & 0,15 \\
\hline \multicolumn{7}{|l|}{ Matéria vegetal } \\
\hline Alga filamentosa & 83 & 280 & 8,46 & 34 & 1870 & 9,03 \\
\hline \multicolumn{7}{|l|}{ Matéria animal } \\
\hline Escama & 80 & 46 & 1,39 & 22 & 126 & 0,39 \\
\hline Peixe & - & - & - & 2 & 280 & 0,08 \\
\hline Sedimento & 65 & 58 & 1,31 & 18 & 186 & 0,48 \\
\hline IAi total vegetais & - & - & 57,6 & - & - & 76,1 \\
\hline IAi total animal & - & - & 42,4 & - & - & 23,9 \\
\hline CI (médio) & - & - & 0,64 & - & - & 0,84 \\
\hline
\end{tabular}

\section{Discussão}

Nesse estudo tivemos como objetivo descrever a dieta de A. janeiroensis, suas respectivas alterações ontogenéticas associadas e as variações espaço-temporais dos itens alimentares consumidos. Observamos que a espécie apresentou hábito onívoro como ingestão de insetos (tanto aquáticos como terrestres), partes de vegetais superiores e algas, com relativa preferência por alimentos de origem vegetal. Classificação semelhante foi adotada por Vilella et al. (2002) e Smith et al. (2004) para algumas espécies de Astyanax por ingerirem grande variedade de itens e terem em insetos e material vegetal os mais importantes itens alimentares da dieta. Outro aspecto observado no presente trabalho foi a ocorrência eventual de alguns itens tais como peixes, escamas e sedimento, evidenciando comportamento de forrageamento oportunista. Esse tipo de comportamento foi amplamente identificado entre algumas espécies de Characidae (sensu Lima et al. 2003) tais como Astyanax e Deuterodon (e.g. Vilella et al. 2002, Lobón-Cerviá \& Bennemann 2000).

Conforme registros anteriores e nossos próprios resultados as espécies de Characidae apresentam ampla variedade de estratégias alimentares sendo comuns espécies herbívoras, insectívoras e/ou onívoras com grande plasticidade associada às variações sazonais ou ontogenéticas (Sabino \& Castro 1990, Kramer \& Bryant 1995, Aranha et al. 1998, Winemiller \& Jepsen 1998, Vitule \& Aranha, 2002, Vilella et al. 2002, Fogaça et al. 2003, Barreto \& Aranha 2006). Esta constatação não surpreende dada a grande diversidade de espécies e formas reunidas no grupo Characidae. A própria situação taxonômica do grupo é um elemento que reforça essa questão, pois a inclusão de vários gêneros incertae sedis, entre eles Astyanax (para maiores detalhes ver Lima et al. 2003), reflete a diversidade de formas e, dessa forma, a amplitude de nichos tróficos apresentados pelas espécies que compõem a família Characidae.

De acordo com Wootton (1998) o crescimento dos peixes é acompanhado por importantes mudanças na dieta e na susceptibilidade à predação, sendo que o primeiro ano é um momento de intenso

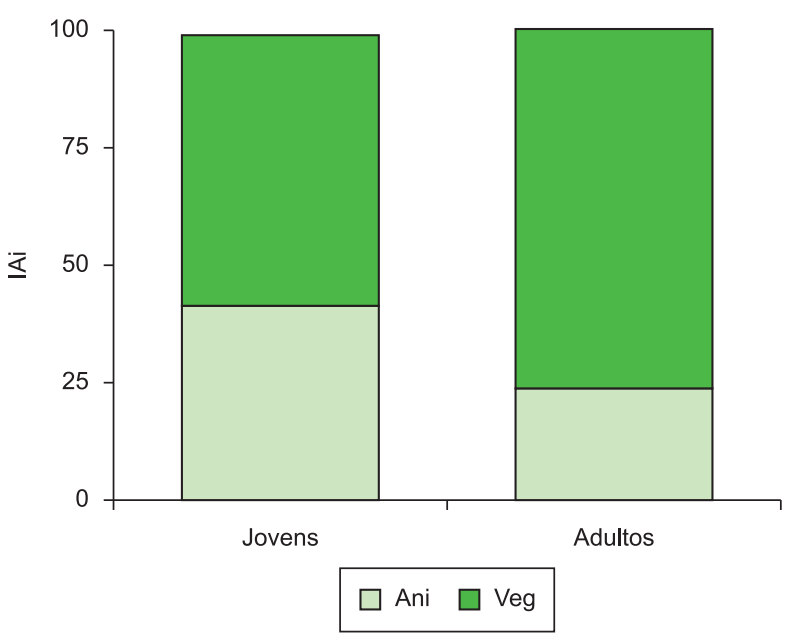

Figura 3. Dados da variação do Índice Alimentar (IAi) dos itens animal vs. vegetal registrados na dieta de exemplares jovens e adultos de Astyanax janeiroensis do Rio Ubatiba.

Figure 3. Alimentary Index (IAi) data variation of animal vs. vegetal food item registered in the diet of juveniles and adult specimens of Astyanax janeiroensis from Ubatiba Stream. 
Tabela 3. Dados da Frequência de Ocorrência (FO), Volume (VO) e Índice Alimentar (IAi) dos diferentes itens alimentares registrados na dieta de exemplares de Astyanax janeiroensis amostrados nas estações seca e chuvosa e nas localidades aberta e fechada do sistema fluvial do Rio Ubatiba.

Table 3. Frequency of occurrence (FO), volume (VO) and Alimentary Index (IAi) of each food items registered in the diet of Astyanax janeiroensis sampled during the dry and rainy seasons at the opened and closed sites at Ubatiba Stream.

\begin{tabular}{|c|c|c|c|c|c|c|c|c|c|c|c|c|}
\hline & \multicolumn{3}{|c|}{ Chuva } & \multicolumn{3}{|c|}{ Seca } & \multicolumn{3}{|c|}{ Aberta } & \multicolumn{3}{|c|}{ Fechada } \\
\hline & FO & VO & IAi & FO & VO & IAi & FO & VO & IAi & FO & VO & IAi \\
\hline \multicolumn{13}{|c|}{ Itens alóctones } \\
\hline \multicolumn{13}{|c|}{ Formas adultas de insetos } \\
\hline Hymenoptera & 63 & 15 & 0,15 & 96 & 575 & 10,83 & 20 & 392 & 2,33 & 21 & 260 & 0,66 \\
\hline Araneae & 10 & 3 & $<0,01$ & - & - & - & - & - & - & 16 & 718 & 1,40 \\
\hline Isoptera & - & - & - & - & - & - & - & - & - & 4 & 6 & $<0,01$ \\
\hline \multicolumn{13}{|c|}{ Matéria vegetal } \\
\hline Sementes & 50 & 44 & 0,32 & 64 & 58 & 0,73 & 12 & 66 & 0,23 & 72 & 60 & 0,53 \\
\hline Restos vegetais & 131 & 1049 & 21,51 & 57 & 26 & 0,29 & 60 & 2722 & 48,44 & 40 & 138 & 0,67 \\
\hline \multicolumn{13}{|c|}{ Itens autóctones } \\
\hline \multicolumn{13}{|c|}{ Formas jovens de artrópodos } \\
\hline Lepidoptera & 10 & 2 & $<0,01$ & 16 & 527 & 1,65 & 4 & 4 & $<0,01$ & 40 & 1346 & 6,56 \\
\hline Diptera & 84 & 419 & 5,52 & 24 & 53 & 0,25 & 20 & 26 & 0,15 & 8 & 1054 & 1,03 \\
\hline Thricoptera & 10 & 3 & $<0,01$ & - & - & - & 4 & 6 & 0,01 & 20 & 110 & 0,27 \\
\hline Coleoptera & 20 & 7 & 0,02 & - & - & - & 8 & 14 & 0,03 & - & - & - \\
\hline Hymenoptera & 30 & 9 & 0,04 & 16 & 17 & 0,05 & 12 & 18 & 0,06 & - & - & - \\
\hline Odonata & 46 & 387 & 2,83 & 8 & 30 & 0,05 & 20 & 774 & 4,59 & 8 & 10 & 0,01 \\
\hline \multicolumn{13}{|c|}{ Formas adultas de artrópodos } \\
\hline Crustácea & 9 & 35 & 0,05 & 70 & 648 & 9,15 & - & - & - & 32 & 142 & 0,55 \\
\hline Fragmento de insetos & 111 & 650 & 11,42 & 129 & 259 & 6,91 & 44 & 760 & 9,92 & 72 & 1058 & 9,27 \\
\hline Hemiptera & 28 & 39 & 0,17 & 10 & 1 & $<0,01$ & 8 & 70 & 0,17 & 4 & 4 & $<0,01$ \\
\hline Coleoptera & 28 & 147 & 0,65 & 23 & 147 & 0,69 & 12 & 294 & 1,05 & 64 & 1596 & 12,44 \\
\hline \multicolumn{13}{|c|}{ Matéria vegetal } \\
\hline Algas filamentosas & 196 & 1831 & 56,31 & 208 & 1650 & 67,33 & 52 & 2080 & 32,11 & 128 & 4240 & 66,08 \\
\hline Matéria animal & 10 & 40 & 0,06 & 32 & 90 & 0,57 & - & - & - & - & - & - \\
\hline Sedimento & 65 & 57 & 0,58 & 48 & 65 & 0,61 & 16 & 102 & 0,48 & 20 & 66 & 0,16 \\
\hline Peixe & 0 & 0 & & 8 & 140 & 0,22 & 2 & 10 & 0,01 & 32 & 60 & 0,23 \\
\hline Escama & 56 & 39 & 0,34 & 71 & 47 & 0,66 & 20 & 70 & 0,42 & 4 & 280 & 0,14 \\
\hline IAi total vegetais & - & - & 78,14 & - & - & 68,35 & - & - & 80,79 & - & - & 67,28 \\
\hline IAi total animal & - & - & 21,84 & - & - & 31,65 & - & - & 19,21 & - & - & 32,71 \\
\hline IAi total autóctone & - & - & 78,00 & - & - & 88,15 & - & - & 49,00 & - & - & 96,73 \\
\hline IAi total alóctone & - & - & 21,98 & - & - & 11,85 & - & - & 51,00 & - & - & 3,26 \\
\hline
\end{tabular}

incremento no tamanho do corpo. O incremento ontogenético do comprimento do intestino em relação ao comprimento corporal tem sido amplamente registrado entre peixes tropicais. Esse processo está relacionado à capacidade de absorção do intestino que, por sua vez, aumenta com o aumento da extensão do mesmo e, entre as espécies onívoras, é fortemente esperada a redução da participação dos itens alimentares de origem animal na medida em que se tornam adultos. Esse processo foi registrado no presente estudo além de ter sido amplamente registrado para outras espécies de Characidae (e.g. Vitule \& Aranha 2002). O uso do Coeficiente Intestinal (CI) pode ser útil para a identificação das variações ontogenéticas refletidas no hábito alimentar (Zavala-Camim 1996). Os valores reduzidos do CI, entre jovens de dada espécie, tem sido atribuídos a dieta predominantemente carnívora (e.g. Sabino \& Castro 1990), enquanto o aumento relativo do intestino dos adultos parece estar associado a grande plasticidade alimentar e uso indiscriminado de itens alimentares de maior tamanho ou de digestão mais difícil, tais como vegetais que possuem paredes celulares rígidas (Sabino \& Castro 1990, Zavala-Camim 1996). Observa-se, então, que muitas espécies iniciam a vida como carnívoras e tendem a ter seus intestinos aumentados em função do crescimento corporal (e.g. Sabino \& Castro 1990, Zavala-Camim 1996). Os valores do CI em associação aos itens alimentares consumidos no presente estudo corroboraram essas proposições e estiveram de acordo com a classificação de Barbieri et al. (1994) sobre as espécies onívoras.

Outra questão importante que permeia os estudos sobre alimentação de peixes se refere às variações temporais e espaciais da dieta. Nossos resultados não evidenciaram diferenças relacionadas às estações seca e chuvosa, possivelmente pela ampla gama de itens alimentares consumidos pela espécie e pela constante disponibilidade de recursos, típica de ambientes tropicais (e.g. Mazzoni et al., 2010). Por outro lado, registramos alterações significativas para a dieta dos exemplares coletados em localidades com e sem cobertura vegetal. As principais diferenças foram relativas a participação dos 
Mazzoni, R. et al.
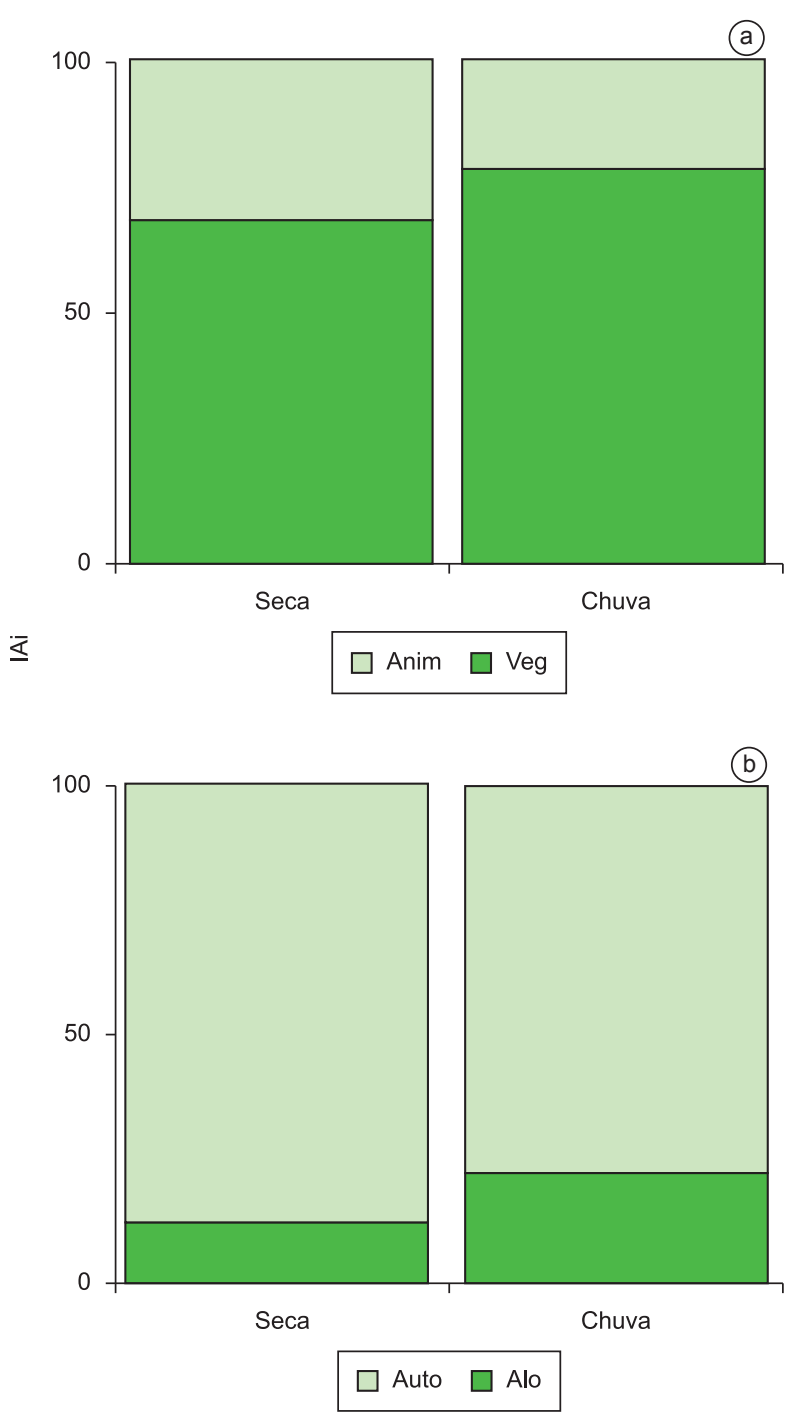

Figura 4. Dados temporais (chuva e seca) da variação do Índice Alimentar (IAi) dos itens animal vs.vegetal (a) e alóctone $v s$. autóctone (b) registrados na dieta de Astyanax janeiroensis do Rio Ubatiba.

Figure 4. Alimentary Index (IAi) data for seasonal (dry and rainy) variation of animal vs. vegetal (a) and allochthonous vs. autochthonous (b) food item registered in the diet of Astyanax janeiroensis from Ubatiba Stream.

itens de origem autóctone e alóctone. Verificamos que a dieta de A. janeiroennsis apresentou predomínio, quase absoluto, de itens autóctones nas localidades fechadas, fato que ressalta a importância dos processos internos na manutenção de sistemas fluviais de baixa ordem. Nossos resultados enfatizam a importância da produção autóctone para a manutenção da espécie no riacho estudado o que nos opõe aos resultados mais comuns sobre ecologia trófica de riachos, que sempre apontam para a importância do aporte de matéria alóctone proveniente da mata ripária (Welcomme 1985, Moulton \& Magalhães 2003, Abilhoa et al. 2009). Apesar de nossos resultados contrastarem com muitos registros da literatura, acreditamos que o comportamento de forrageamento da espécie, amplamente apoiado
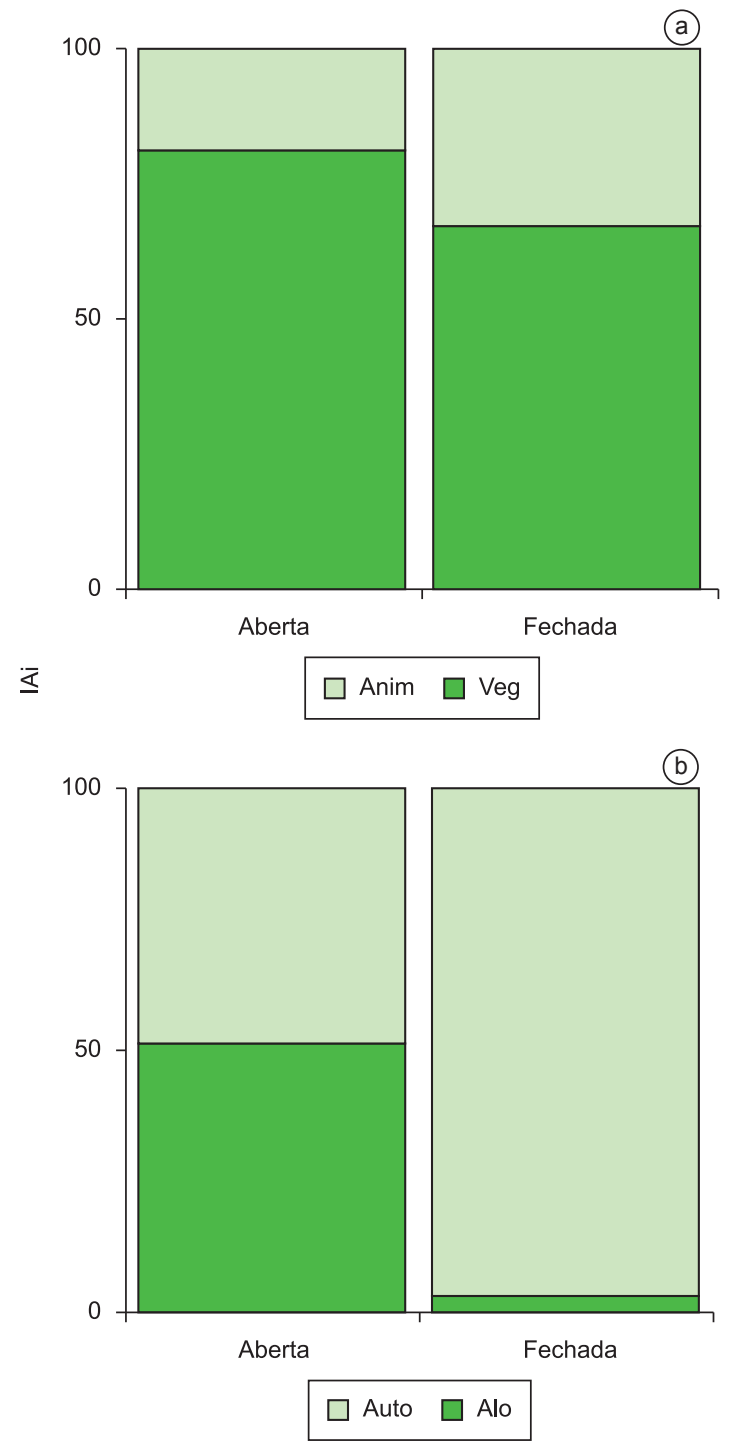

Figura 5. Dados espaciais (localidades aberta e fechada) da variação do Índice Alimentar (IAi) dos itens animal vs.vegetal (a) e alóctone vs. autóctone (b) registrados na dieta de Astyanax janeiroensis do Rio Ubatiba.

Figure 5. Alimentary Index (IAi) data for spatial (opened and closed sites) variation of animal vs. vegetal (a) and allochthonous vs. autochthonous (b) food item registered in the diet of Astyanax janeiroensis from Ubatiba Stream.

no oportunismo, explique os resultados encontrados e não invalidem a importância da vegetação ripária para a manutenção da integridade dos riachos de baixa ordem.

\section{Agradecimentos}

Agradecemos aos membros do Laboratório de Ecologia de Peixes da UERJ pelo auxílio no trabalho de campo e triagem do material e a Javier Lobón-Cerviá pelas sugestões na primeira versão do manuscrito. Esse trabalho foi parte do trabalho de iniciação científica de LLN. Foi financiado pela FAPERJ - E-26/171.231/98-APQ1 e CNPQ - 479426/01-5. RM possui bolsa de pesquisa do CNPq 301433/2007-0. 


\section{Referências Bibliográficas}

ABILHOA, V., BASTOS, L.P. \& WEGBECHER, F. 2007. Feeding habits of Rachoviscus crassiceps (Teleostei: Characidae) in a coastal Atlantic rainforest stream, southern Brazil. Ichthyol. Exp. Freshw. 18(3):227-232

ABILHOA, V., BORNATOWSKI, H. \& OTTO, G. 2009. Temporal and ontogenetic variations in feeding habits of Hollandichthys multifasciatus (Teleostei: Characidae) in coastal Atlantic rainforest streams, southern Brazil. Neotrop. Ichthyol. 7(3):415-420.

ANGELESCU, V. \& GNERI, F.S. 1949. Adaptaciones del aparato digestivo al regimen alimentício em algunos peces del Rio Uruguay del Rio de la Plata. Rev. Inst. Nac. Inv. Ciênc. Nat. 2:159-272.

ARANHA, J.M.R., TAKEUTI, D.F. \& YOSHIMURA, T.M. 1998. Habitat use and food partitioning of the fishes in a coastal stream of Atlantic Forest, Brazil. Rev. Biol. Trop. 46(4):951-959.

BARBIERI, G., PERET, A.C. \& VERANI, J.R. 1994. Notas sobre a adaptação do trato digestivo ao regime alimentar em espécies de peixes da região de São Carlos (SP). I. Quociente intestinal. Braz. J. Biol. 54(1):63-69.

BARRETO, A.P. \& ARANHA, J.M.R. 2006. Alimentação de quatro espécies de Characiformes de um riacho da Floresta Atlântica, Guaraqueçaba, Paraná, Brasil. Rev. Brasil. Zool. 23(3):779-788.

BORROR, D.J. \& DELONG, D.M. 2005. Study of insects. Thomson Learning Academic Resource Center, Belmont.

CARVALHO, A.L. 1989. Description of the larva of Neuraeschna costalis (Burmeister), with notes of its biology, and a key to the genera of Brazilian Aeshnidae Larvae (Anisoptera). Odonatologica 18(4):325-332.

CARVALHO, A.L. 1992. Revalidation of the genus Remartinia Navás, 1911, with the description of a new species and a key to the genera of Neotropical Aeshnidae (Anisoptera). Odonatologica 21(3):289-298.

CARVALHO, A.L., WERNECK-DE-CARVALHO, P.C. \& CALIL, E.R. 2001 Description of the larvae of two species of Dasythemis Karsch, with a key to the genera of Libellulidae occurring in the states of Rio de Janeiro and São Paulo, Brazil (Anisoptera). Odonatologica 31(1):23-33.

CASSATI, L. 2002. Alimentação dos peixes em um riacho do Parque Estadual Morro do Diabo, bacia do Alto Paraná, sudeste do Brasil. Biota Neotrop. 2(2):01-14.

COSTA, W.J.E.M. 1987. Feeding habits of a fish community in a tropical coastal stream, Rio Mato Grosso, Brazil. Stud. Neot. Fauna Environ. 22(2):145-153

DUFECH, A.P.S., AZEVEDO, M.A. \& FIALHO, C.B. 2003. Comparative dietary analysis of two populations of Mimagoniates rhocharis (Characidae: Glandulocaudinae) from two streams of Southern Brazil. Neotrop. Ichthyol. 1(1):67-74

FOGAÇA, F.N.O., ARANHA, J.M.R. \& ESPER, M.L.P. 2003. Ictiofauna do Rio do Quebra (Antonina, PR, Brasil): ocupação espacial e hábito alimentar. Interciencia 28:168-173.

GERKING, S.D. 1994. Feeding ecology of fishes. Academic Press, San Diego.

HAHN, N.S., FUGI, R., ALMEIDA, V.L.L., RUSSO, M.R. \& LOUREIRO, V.E. 1997. Dieta e atividade alimentar de peixes do reservatório de Segredo. In Reservatório de Segredo - bases ecológicas para o manejo (A.A. Agostinho \& L.C. Gomes eds.). Eduem/Nupelia, Maringá, 387p.

HYSLOP, E.J. 1980. Stomach contents analysis - a review of methods and their application. J. Fish Biol. 17:411-429.

KAWAKAMI, E. \& VAZZOLER, G. 1980. Método gráfico e estimativa de índice alimentar aplicado no estudo da alimentação de peixes. Bol. Inst. Oceon. 29(2):205-207.

KRAMER, D.L. \& BRYANT, M.J. 1995. Intestine length in the fishes of a tropical stream: 2. Relationships to diet: the long and short of a convoluted issue. Env. Biol. Fish. 42:129-141.

LIMA, F.C.T., MALABARBA, L.R., BUCKUP, P.A., PEZZI DA SILVA, J.F., VARI, R.P., HAROLD, A., BENINE, R., OYAKAWA, O.T., PAVANELLI, C.S., MENEZES, N.A., LUCENA, C.A.S., MALABARBA, M.C.L.S., LUCENA, Z.M.S., REIS, R.E., LANGEANI, F., CASSATI, L. \&
BERTACO, V.A. 2003. Genera Incertae Sedis in Characidae. In Checklist of the Freshwater Fishes of South and Central America (R.E. Reis, S.O. Kullander \& C.J. Ferraris-Jr eds.). EDIPUCRS, Porto Alegre, p. $106-168$.

LOBÓN-CERVIÀ, J. \& BENNEMANN, S. 2000. Temporal trophic shifts and feeding diversity in two sympatric, neotropical, omnivorous fishes: Astyanax bimaculatus and Pimelodus maculatus in Rio Tibagi (Paraná, Southern Brazil). Arch. Hydrobiol. 149(2):205-306.

LOWE-MCCONELL, R.H. 1991. Ecological Studies in Tropical Fish Communities. Cambridge University Press, Cambridge.

MATTHEWS, W.J. 1998. Patterns in Freshwater Fish Ecology. Chapman \& Hall, London.

MAZZONI, R. \& COSTA, L.D.S. 2007. Feeding Ecology of Stream-Dwelling Fishes from a Coastal Stream in the Southeast of Brazil. Brazil. Arch. Biol. Technol. 50(4):627-635.

MAZZONI, R. \& LOBÓN-CERVIÁ, J. 2000. Longitudinal structure, density and production rates of a neotropical stream fish assemblage: the river Ubatiba in the Serra do Mar, southeast Brazil. Ecography 23(5):588-602.

MAZZONI, R., ARAUJO, R.S., SANTOS, G.C.T. \& IGLESIAS-RIOS, R. 2010. Feeding ecology of Phalloceros anisophallos (Osteichthyes, Cyprinodontiformes) from Andorinha Stream, Ilha Grande, Brazil. Neotrop. Ichthyol. 8(1):179-182.

MAZZONI, R., FRENERICH-VERANI, N. \& CARAMASCHI, E.P. 2000. Electrofishing as a sampling technique for coastal stream fish populations in the Southeast of Brazil. Rev. Brasil. Biol. = Brazil. J. Biol. 60(2):205-216

MAZZONI, R., IGLESIAS-RIOS, R. \& SCHUBART, S.A. 2004. Longitudinal segregation of Astyanax janeiroensis in Rio Ubatiba: a Neotropical stream of south-east Brazil. Ecol. Freshw. Fish 13:231-234.

MAZZONI, R., MENDONÇA, R.S. \& CARAMASCHI, E.P. 2006. Reproductive biology of Astyanax janeiroensis (Osteichthyes, Characiidae) from the Ubatiba river, Maricá, RJ, Brasil. Braz. J. Biol. 65(4):643-649.

MELO, GAS. 2003. Manual de identificação de crustácea decapoda de água doce do Brasil. Loyola, São Paulo.

MOULTON, T.P. \& MAGALHÃES, S.A.P. 2003. Responses of leaf processing to impacts in streams in Atlantic Rain Forest, Rio de Janeiro, Brazil - A test of the biodiversity-ecosystem function relationship? Brazil. J. Biol 63:87-95.

OLIFIERS, M.H., DORVILLÉ, L.F.M. \& NESSIMIAN, J.L. 2004. A key to Brazilian genera of Plecoptera (Insecta) based on nymphs. Zootaxa 651:1-15.

PASSOS, M.I.S., NESSIMIAN, J.L. \& FERREIRA Jr., N.F. 2007. Chaves para identificação dos gêneros de Elmidae (Coleóptera) acorrentes no estado do Rio de Janeiro, Brasil. Rev. Brasil. Ent. 51(1):42-53.

PES, AMO., HAMADA, N. and NESSIMIAN, JL. 2005. Chaves de identificação de larvas para famílias e gêneros de Trichoptera (Insecta) da Amazônia Central, Brasil. Rev. Brasil. Ent. 49(2):181-204

PRINGLE, C.M. \& HAMAZAKI, T. 1998. The role of omnivory in a neotropical stream: separating diurnal and nocturnal effects. Ecology 79:269-280.

REZENDE, C.F. \& MAZZONI, R. 2006a. Disponibilidade e uso de recursos alóctones por Bryconamericus microcephalus (Miranda-Ribeiro) (Actinopterygii, Characidae), no córrego Andorinha, Ilha Grande - Rio de Janeiro, Brasil. Rev. Brasil. Zool. 23(1):218-222.

REZENDE, C.F. \& MAZZONI, R. 2006b. Contribuição da matéria autóctone e alóctone para a dieta de Bryconamericus microcephalus (MirandaRibeiro) (Actinopterygii, Characidae), em dois trechos de um riacho de Mata Atlântica, Rio de Janeiro, Brasil. Rev. Brasil. Zool. 23(1):58-63.

SABINO, J. \& CASTRO, R.C. 1990. Alimentação, período de atividade e distribuição espacial dos peixes de um riacho de floresta atlântica (sudeste do Brasil). Rev. Brasil. Zool. 50(1):23-36. 
SALLES, F.F., DA-SILVA, E.R., SERRÃO, J.E. \& FRANCISCHETTI, C.N. 2004. Baetidae (Ephemeroptera) na região Sudeste do Brasil: Novos registros e chave para os gêneros no estágio ninfal. Neotrop. Entomol. 33(5):725-735.

SMITH, W.S., PEREIRA, C.C.G.F. \& ESPÍNDOLA, E.L.G. 2004. Hábitos alimentícios de nueve especies de peces del embalse de Três Irmãos, São Paulo, Brasil. Univers. Cienc. 1: 33-38.

VILELLA, F.S., BECKER, F.G. \& HARTZ, S.M. 2002. Diet of Astyanax species (Teleostei, Characidae) in an Atlantic Forest River in Southern Brazil. Brazil. Arch. Biol. Technol. 45(2):223-232.

VITULE, J.R.S. \& ARANHA, J.M.R. 2002. Ecologia alimentar do lambari, Deuterodon langei Travassos, 1957 (Characidae, Tetragonopterinae), de diferentes tamanhos em um riacho da Floresta Atlântica, Paraná (Brasil). Acta Biol. Paran. 31:137-150.
WELCOMME, R.L. 1985. Ontogenetic diet shifts and resource partitioning among piscivorous fishes in the Venezuelan llanos. Env. Biol. Fish 26(1):177-199.

WINEMILLER, K.O. \& JEPSEN, D.B. 1998. Effects of seasonality and fish movement on tropical river food webs. J. Fish Biol. 53(3):267-296.

WOOTTON, R. 1998. Ecology of teleost fishes. Kluwer Academic Publishers, London.

ZAVALA-CAMIN, L.A. 1996. Alimentação de peixes. In Situação atual e perspectivas da ictiologia no Brasil (A.A. Agostinho \& E. Beneditocecílio, eds.). Editora UEM, Maringá, p. 14-18.

Recebido em 05/04/2010

Versão reformulada recebida em 09/06/2010

Publicado em 06/07/2010 